\title{
Effective nivolumab sequential thoracic radiotherapy in elderly patients with advanced squamous cell lung cancer: did radiation therapy play a role? A case report
}

This article was published in the following Dove Press journal: OncoTargets and Therapy

\author{
Grazia Lazzari' \\ Angela Terlizzi ${ }^{2}$ \\ Giovanna Porrazzo' \\ Salvatore Devicienti' \\ Francesco Perri ${ }^{3}$ \\ Giuseppina Della \\ Vittoria Scarpati ${ }^{3}$ \\ Giovanni Silvano' \\ 'Radiation Oncology Unit, San \\ Giuseppe Moscati Hospital, Taranto, \\ Italy; ${ }^{2}$ Physics Department, San \\ Giuseppe Moscati Hospital, Taranto, \\ Italy; ${ }^{3}$ Medical Oncology Department, \\ San Giuseppe Moscati Hospital, \\ Taranto, Italy
}

\begin{abstract}
Advanced squamous cell lung carcinoma in elderly patients has a limited chance of cure with first, second line chemotherapy and radiotherapy. Radiotherapy in advanced nonsmall-cell lung cancer can be used with curative intent for localized or oligometastatic disease using standard or altered fractionations. Current evidence indicates that radiotherapy via diverse cascade mechanisms is able to invoke both local and systemic immunoresponses promoting tumor cell death through an in situ vaccination effect. Moreover, the advancement in immunotherapies is changing the scenario. The combination of radiotherapy and immunotherapy could be a crucial strategy to overcome cancer immunoresistance and improve patient survival, as we found in this case report of an elderly, refractory advanced lung cancer patient who has achieved complete remission after this therapeutic combination.
\end{abstract}

Keywords: immunotherapy, anti PD-1 inhibitor, immuno-checkpoint, radiotherapy, in situ vaccination

\section{Introduction}

Non-small-cell lung cancer (NSCLC) accounts for over $80 \%$ of all lung cancers. Approximately $75 \%$ of all NSCLC cases are diagnosed at advanced stage with a median survival of 4-5 months after diagnosis and 10\% of 1-year estimated survival. ${ }^{1}$ Furthermore, nearly $50 \%$ of all cases of lung cancer occur in the elderly subpopulation aged over 70 years, in which physiological changes together with comorbidities exert a negative impact on systemic therapy tolerability and overall outcome. ${ }^{2}$ Among histologies, squamous cell carcinoma represents approximately $30 \%$ of all NSCLC, the management of which, with first and second line chemotherapy, is difficult in elderly patients. ${ }^{3}$ A combination of two drugs in first line chemotherapy - cisplatin plus vinorelbine or weekly nab-paclitaxel with carboplatin - has shown superior efficacy over monotherapy in fit elderly patients, although this benefit occurs at the expense of the patient in terms of added toxicities. ${ }^{4,5}$ But for second line therapy little therapeutic progress has been made since the approval of docetaxel in $1999 .{ }^{6}$ Since then, no more effective agents have been found due to toxicity and lack of specific genetic alterations in squamous cell lung carcinoma.

Radiotherapy is acknowledged as an important partner in lung cancer treatment, but in elderly patients with advanced stage squamous cell lung carcinoma, it is not always used with curative intent. However, evidence suggests that cases with oligometastatic
Correspondence: Grazia Lazzari Radiation Oncology Unit, San Giuseppe Moscati Hospital, Road to Martina Franca, Taranto 74100 , Italy

Tel +390994585829

Email lazzarigrazia@gmail.com 
disease responding to systemic chemotherapy may be selected for curative radiotherapy. ${ }^{7}$

Advances in cancer immunotherapy and emerging knowledge of the immunogenic power of radiation might change the scenario and promote cure.

The cancerogenesis process seems to be based on altered cancer immuno-surveillance as a result of tumor cells escaping immunity, ${ }^{8}$ and there is an increased body of evidence showing that radiotherapy enhances immune responses against cancer. ${ }^{9}$ A combination of several immunotherapeutic strategies and radiation may be able to reshape the tumor's microenvironment toward the inhibition of tumor-induced immunosuppression, leading to the immunogenic death of cancer cells. ${ }^{10}$

Promising effects are coming from therapies directed against cancer immunoresistance mechanisms such as programmed cell death protein-1 (PD-1) signaling or its ligands (PD-L1, PD-L2) and CTLA-4. ${ }^{11}$ Among them, one important mechanism involved in tumor cells' escape recognition and elimination by the immune system is the interaction of PD-1/PD-L1. ${ }^{12}$ These proteins are prevalently expressed in NSCLC and have been found to down-regulate T cell activation and promote tumor "immune escape". ${ }^{13}$ Studies have demonstrated that the inhibition of the PD-L1/PD-1 pathway is effective for refractory solid tumors, but the efficiency of this treatment related to the tumor PD-1/PD-L1 expression level remains unclear and controversial. ${ }^{14}$ Experimental preliminary results suggested that PD-L1 positivity in the tumor specimen may correlate with response to treatment with anti PD-1 inhibitors. ${ }^{15,16}$ Additionally, a positive clinical outcome seems to be related to tumor-infiltrating lymphocytes (TILs) in the specimen. ${ }^{17}$ Interestingly, TILs have been found to have a prognostic and predictive value in patients with advanced NSCLC treated with platinum-based chemotherapy. ${ }^{18}$

Current evidence indicates that radiotherapy can, via diverse cascade mechanisms, invoke both local and systemic immunoresponses promoting tumor-cell death through an in situ vaccination effect. ${ }^{19}$ These immunostimulatory properties have recently generated a widespread interest in light of the preclinical and clinical evidence of the "abscopal effect" in which localized hypofractionated radiotherapy has induced an antitumor immune response mediating the regression of non-irradiated metastases. ${ }^{20}$

Combining radiotherapy and immunotherapy could be a crucial strategy to overcome cancer immunoresistance and improve patient survival, as we have supposed for this case report. ${ }^{21}$

\section{Case presentation}

Written informed consent was obtained from the patient for publication of this case report and any accompanying images. A copy of the written consent is available for review with the Editor in Chief of this journal.

In January 2015, an 80-year old Caucasian male patient came to our attention suffering from cough with blood in sputum, dyspnea, and moderate weight loss. He informed us that he had been a smoker of 20 cigarettes per day but stopped 10 years ago and he did not report alcohol consumption. At clinical examination he showed an ECOG performance status of 1 , while no palpable lymph nodes in the neck and supraclavicular fossa were found. The chest X-ray recorded a mass in the right lung measuring at least $3 \mathrm{~cm}$ with a nodular lesion in the posterior side of the same lobe plus a nodule in the left lung's upper lobe. The total body computed tomography (CT) confirmed a contrast enhancing irregular mass measuring $35 \times 25 \mathrm{~mm}$ in the upper lobe of the right lung with the nodule in the posterior side of the same lobe near the pulmonary fissure measuring $10 \mathrm{~mm}$. A nodule in the upper posterior lobe of the contralateral lung, of $27 \mathrm{~mm}$, was also identified (Figure 1).

Subsequently, PET confirmed the suspicion of malignancy in both lungs: the large right lesion showed a high uptake with standardized uptake value 14.1, while the smaller one had a 2.1 SUV; the left nodule had a SUV 9.1. Brain Magnetic Resonance Imaging (MRI) scan was negative. Stage of disease was T3 N0 M1a (Stage IV) according to American Joint Committee on Cancer (AJCC) seventh edition (Figure 2).

A bronchoscopy confirmed the presence of a stenotic exophytic lesion arising from the mucosa of the sub-segmentary upper right bronchus. A biopsy on the nodule of the left lung and involved bronchus was done during bronchoscopy; the histopathologic examination identified NSCLC in both specimens.

Histopathologic examination identified a poorly differentiated (grade 3) squamous cell carcinoma associated with an infiltration of lymphocytes and macrophages distributed in the specimen. TILs were assessed only by H\&E staining but CD3/CD4-CD8 subpopulations were not specified. Immunohistochemistry was employed to detect biological features such as TIF-1, CK7, and p63. Consequently, TIF-1 and CK7 negativity and p63 positivity were found.

Indirect immunoperoxidase procedures were carried out to assess the PD-1 and PD-L1 expression and, as a result, a moderate staining (2+) in more than $5 \%$ of tumor cells was observed.

Given the stage of the disease, the squamous histology, age $>70$ years, and performance status 1 typical of 

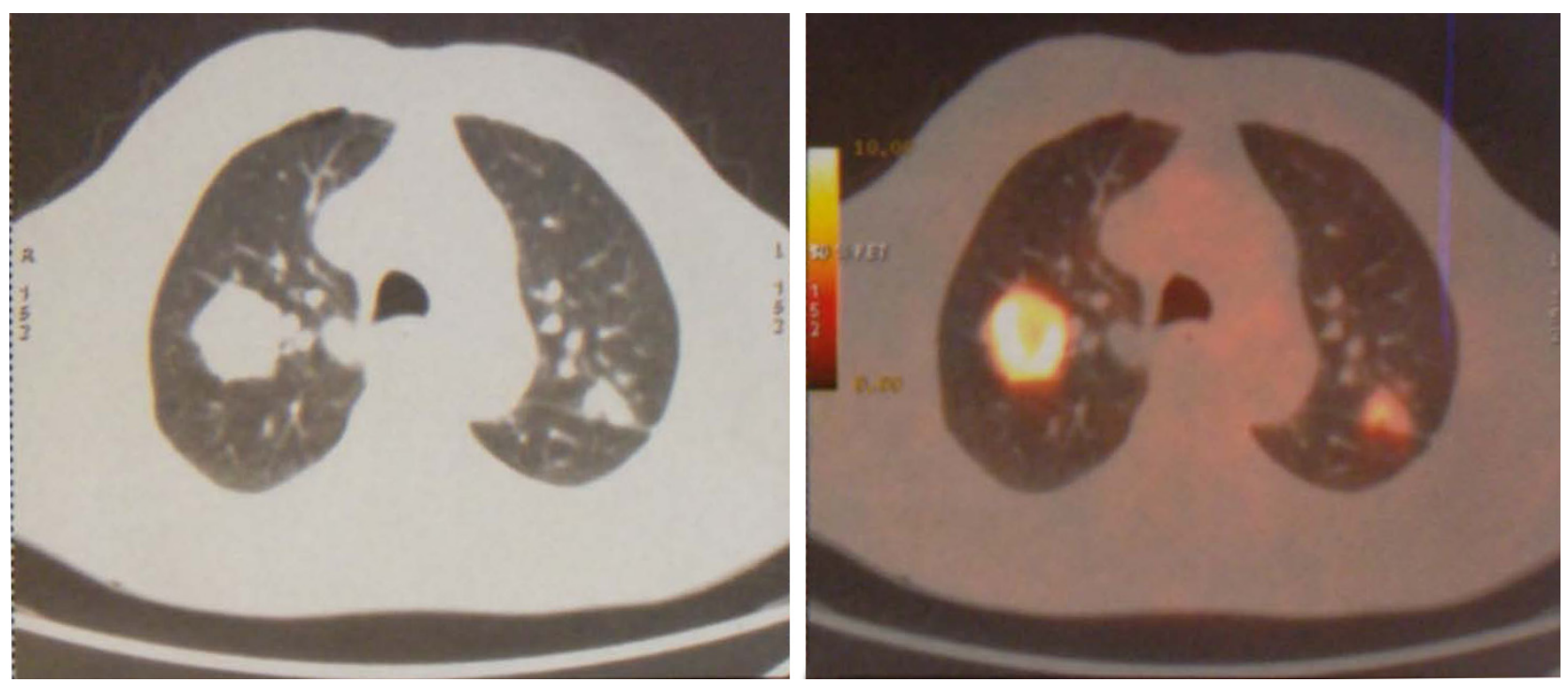

Figure I First diagnosis with CT and PET of the chest.

Note: The image shows the primary lesion with a nodule in the right lung and another nodule in the left lung.

a fit elderly patient, cisplatin-containing chemotherapy treatment scheme was prescribed. The chosen scheme consisted of cisplatin $50 \mathrm{mg} / \mathrm{m}^{2}$ on day 1 plus intravenous vinorelbine $22.5 \mathrm{mg} / \mathrm{m}^{2}$ on days 1 and 8 every 21 days for four cycles. Only two cycles were delivered due to acute severe toxicity - Grade 2 anemia and G3 febrile neutropenia requiring erythropoietin for 2 months and G-CSF for 3-4 days and antibiotics. After hematologic recovery, the patient continued to receive intravenous vinorelbine, as monochemotherapy, $25 \mathrm{mg} / \mathrm{m}^{2}$ on days $1-8$ every 3 weeks for four cycles. Acute toxicity was mild - G2 thrombocytopenia after the last cycle and constipation.
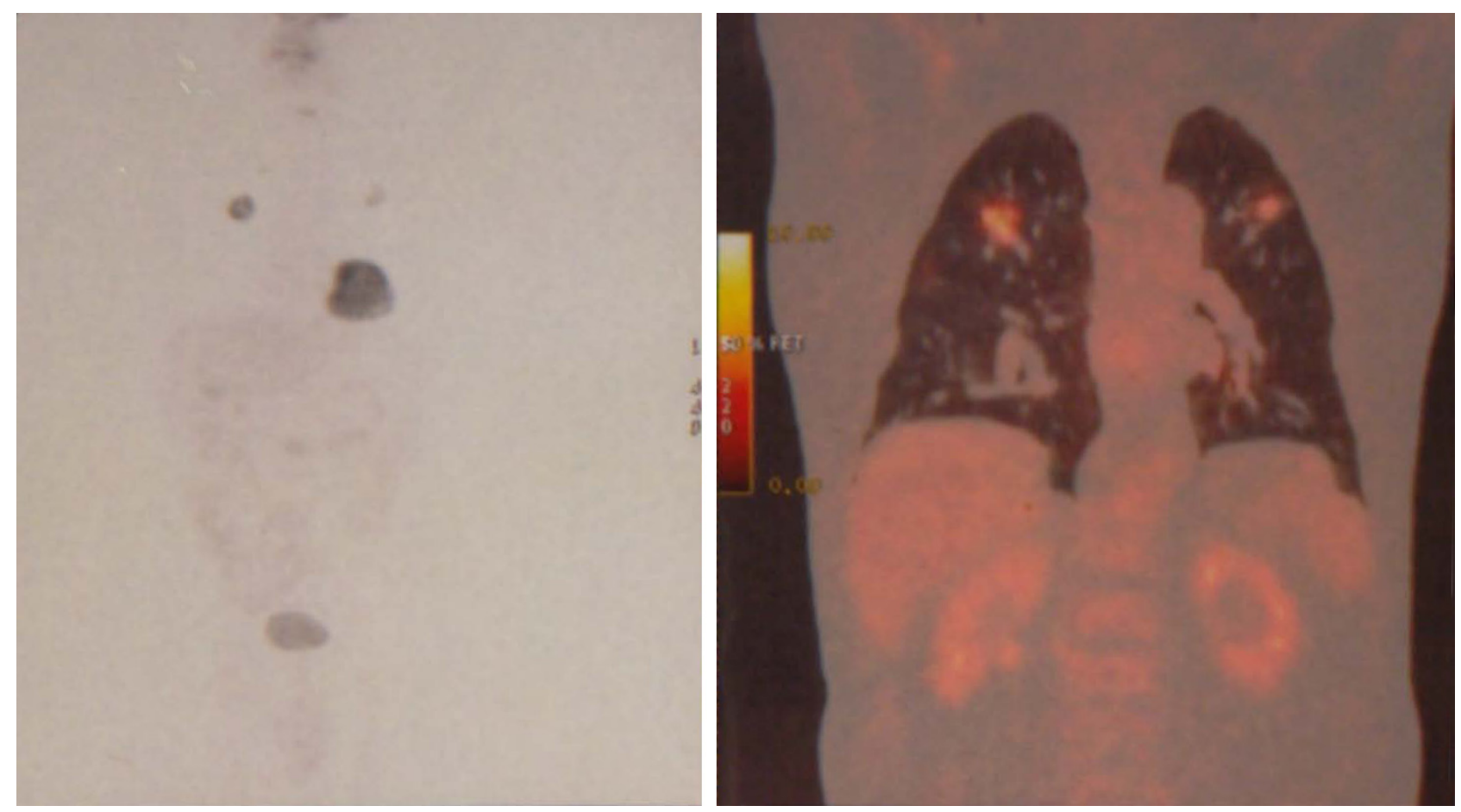

Figure 2 PET at first staging.

Note: The images confirm the nodular lesions in both lungs as stage T3 NO Mla. 
One month after the last vinorelbine delivery, restaging $\mathrm{PET} / \mathrm{CT}$ was performed, only a complete remission of the two pulmonary nodules was observed, while the right intrapulmonary lesion was increased measuring $45 \mathrm{~mm}$ with an avid fluorodeoxyglucose (FDG) uptake with an SUV 18. The disease was downstaged to yT2a N0 M0 (stage IB) due to disappearance of the two nodules (Figure 3).

On the basis of these results, the patient was considered eligible for locoregional treatment with thoracic radiotherapy. Respiratory function tests ( $\mathrm{FEV}_{1}$ and DLCO) were required to assess the lung capacities and diffusion. Spirometry recorded a restrictive respiratory impairment with an $\mathrm{FEV}_{1} 2.36 \mathrm{~L}$; the alveolar-capillary diffusion was low with a DLCOc/VA $0.54 \mathrm{mmol} / \mathrm{min} / \mathrm{kPa} / \mathrm{L}$ (47\%) due to interstitial damage. 3D conformal radiotherapy was delivered using five multileaf collimator customized isocentric fields and MV 10 photon beams. The planning target volume (PTV) consisted of the intrapulmonary post-chemotherapy right lesion as gross tumor volume (GTV) with an isotropic margin of $1.8 \mathrm{~cm}$. The prescribed dose was 66 Gy with standard fractionation ( $2 \mathrm{~Gy} / \mathrm{fr}$ ). To minimize the risk of radiation pneumonitis, in the histogram dose volume plot (DVH) the constraint limit for the whole lung as the sum of both lungs was respected with V20 $=25 \%$; V10 $=45 \%$; V $13=38 \%$, V5 $=50 \%$ and mean lung dose $=12 \mathrm{~Gy}$. Concurrent chemoradiotherapy was excluded due to initial stage, age, and previously recorded chemotherapy-induced toxicity.

Acute radiation toxicity was moderate - G2 dyspnea occurring during the 4 th week of treatment. One month after completion radiotherapy, despite the optimal lung DVH as mentioned previously, the patient developed symptomatic acute febrile grade one radiation pneumonitis. Steroids and antibiotics were effective. At second restaging 1 month after pneumonitis was resolved, a new CT scan showed only a modest reduction of the right pulmonary mass with a high contrast enhancement surrounded by interstitial fibrosis. The PET after 3 months of radiotherapy showed a metabolically active right pulmonary lesion with avid FDG uptake (SUV 14) (Figure 4). The patient was diagnosed with a refractory tumor. At that time the patient complained of moderate exertional and at-rest dyspnea, fatigue due to anemia (with $\mathrm{Hb} 10 \mathrm{~g} / \mathrm{dL}$ ), and weight loss of more than $5 \mathrm{~kg}$. At clinical examination the patient
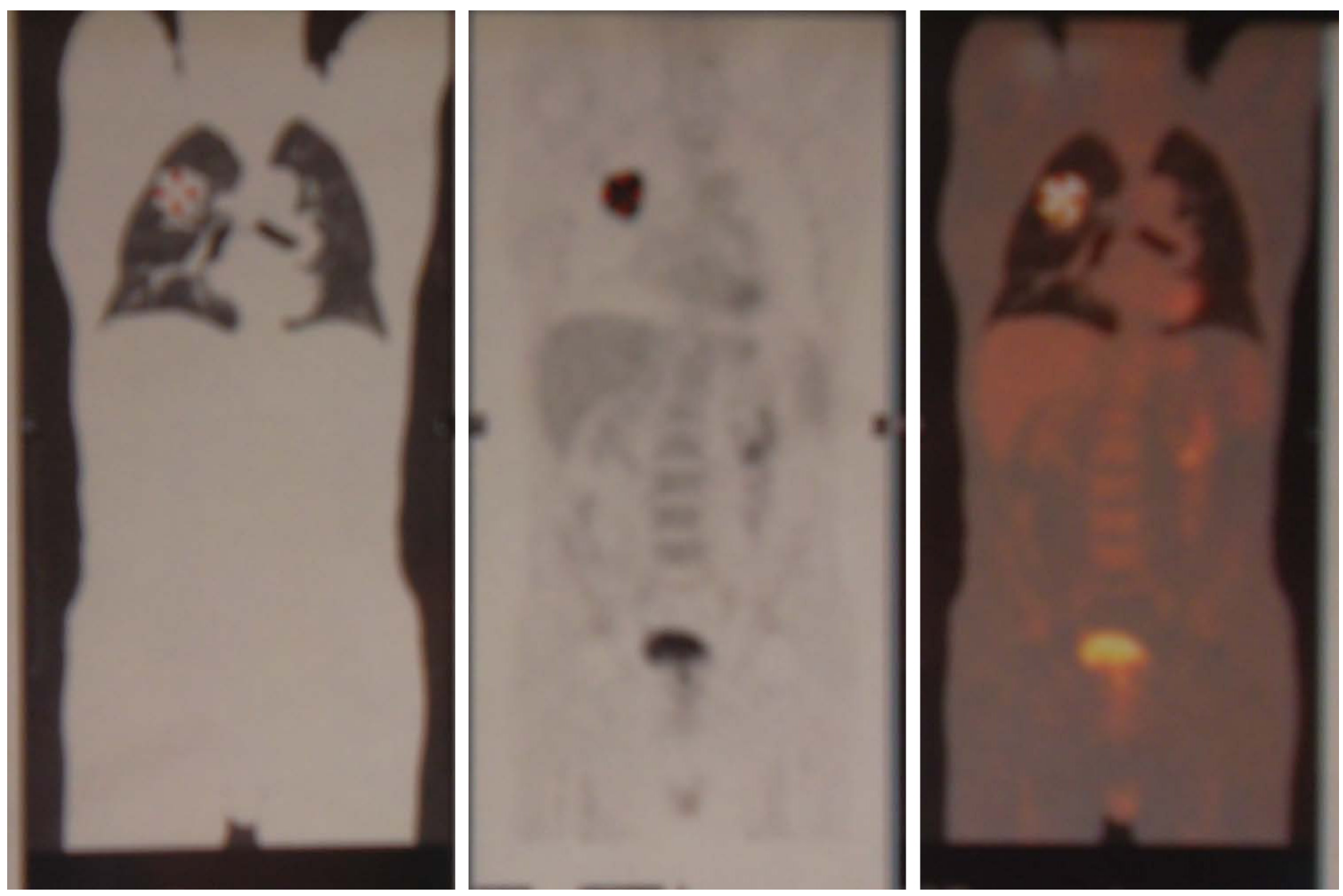

Figure 3 PET at first restaging after chemotherapy.

Note: The disease is downstaged with persistence of primary tumor in right lung: the stage is yT2 NO M0. 

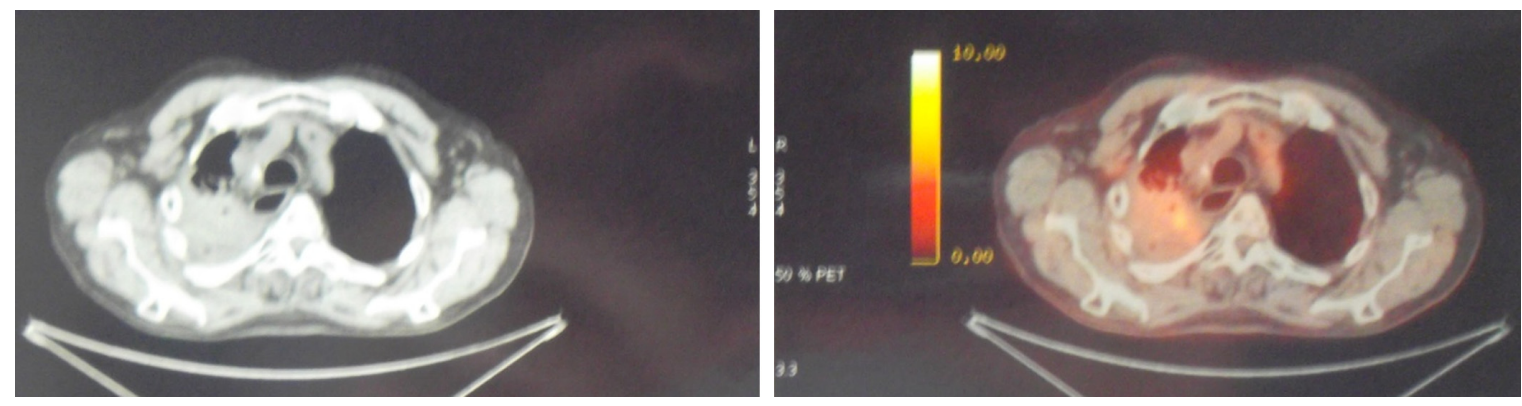

Figure $4 \mathrm{CT} / \mathrm{PET}$ at second restaging after thoracic radiotherapy (66 Gy dose delivered). Note: The findings of post-radiation pneumonitis and persistent disease are recorded.

appeared more frail, pale, and unfit for further combination chemotherapy. The stage of disease remained as yT2 N0 M0. As second line of systemic therapy, we chose to start immunotherapy on the basis of the previous PD-1 determination of the biopsy specimen. Nivolumab monotherapy $3 \mathrm{mg} / \mathrm{kg}$ intravenously every 3 weeks for a total of eight cycles was prescribed and started 4 months after radiation completion. Pruritus was the main acute side effect, no other adverse effects were recorded. During this therapy the performance status and fatigue were ameliorated with a hemoglobin level increase to $12 \mathrm{~g} / \mathrm{dL}$ and a good weight gain of more than $10 \mathrm{~kg}$. After six cycles of nivolumab, the patient underwent total body CT restaging which showed complete resolution of the right pulmonary lesion consisting of a tissue loss with a cavitation instead of the avidly irradiated mass surrounded by an extended pulmonary fibrosis in the of extended pulmonary in the medio-apical part of the right lung.

After eight cycles the new PET confirmed the complete resolution of the tumor, as well as no FDG uptake (Figure 5). After 24 months (the last nivolumab delivery), the PET and CT were still negative for local or systemic disease recurrence. The patient, who is now 83 years of age, has had no disease recurrence (as of December 2017), with a good PS, and has regained quality of life.

\section{Discussion}

NSCLC is the most common cancer in the world and the leading cause of cancer-related deaths in Western countries. ${ }^{22}$ Approximately one third of lung cancers are diagnosed in patients older than 75 years and the majority of them already have metastatic disease. ${ }^{2}$ Palliation is the main or the only therapeutic option. In regard to an effective systemic approach to this population, some Phase II randomized trials confirmed the cisplatin combination plus vinorelbine as a feasible and active treatment in elderly patients with advanced NSCLC. ${ }^{4}$ Vinorelbine alone or with cisplatin for physically fit, chemo-naïve NSCLC patients older than 70 years, has been defined effective with a better response rate and a longer median time to disease progression. However, both statistically significantly higher toxicity and no survival advantage for the combination treatment have been observed..$^{23}$

Our patient was treated according to this currently available evidence-based therapy and developed acute hematologic toxicity as expected, and a poor outcome. The only evaluable positive effect was downstaged disease which consisted of the resolution of the pulmonary nodules, which meant he was eligible for thoracic radiotherapy. ${ }^{7}$ However, radiotherapy did not yield a curative result. This scenario and the conclusions to be drawn could be discouraging because an elderly patient with advanced squamous lung cancer, whose disease does not respond to first line chemotherapy and curative radiotherapy, has limited chances of cure. Finally, the proven existence of altered immunosurveillance in cancer development and the availability of immunotherapy could reshape this scenario, as we have hypothesized in this case.

An old hypothesis of active immunosurveillance that protects against cancer has been revalued ${ }^{24}$ and most recently refined into the process termed "cancer immunoediting", in which tumor cells and immune cells interact within its microenvironment through three $\mathrm{E}$ phases (elimination, equilibrium, escape $)^{25}$ and by prevalence of one of them, the tumor's fate depends. ${ }^{26}$ Research has revealed the ability of tumors to escape the immune system through the activation of distinct pathways including endogenous "immune check points" such as targeted proteins, eg, PD-1, PD-L1, and CTLA-4, which exert a negative regulation on immune anticancer response, permitting cancer progression and metastasis. ${ }^{27}$ Advances in immunotherapy have resulted in therapies against these immune check points, the so called immune check point inhibitors, ${ }^{28}$ which have achieved promising results in refractory metastatic solid tumor-affected patients. $^{29}$ 

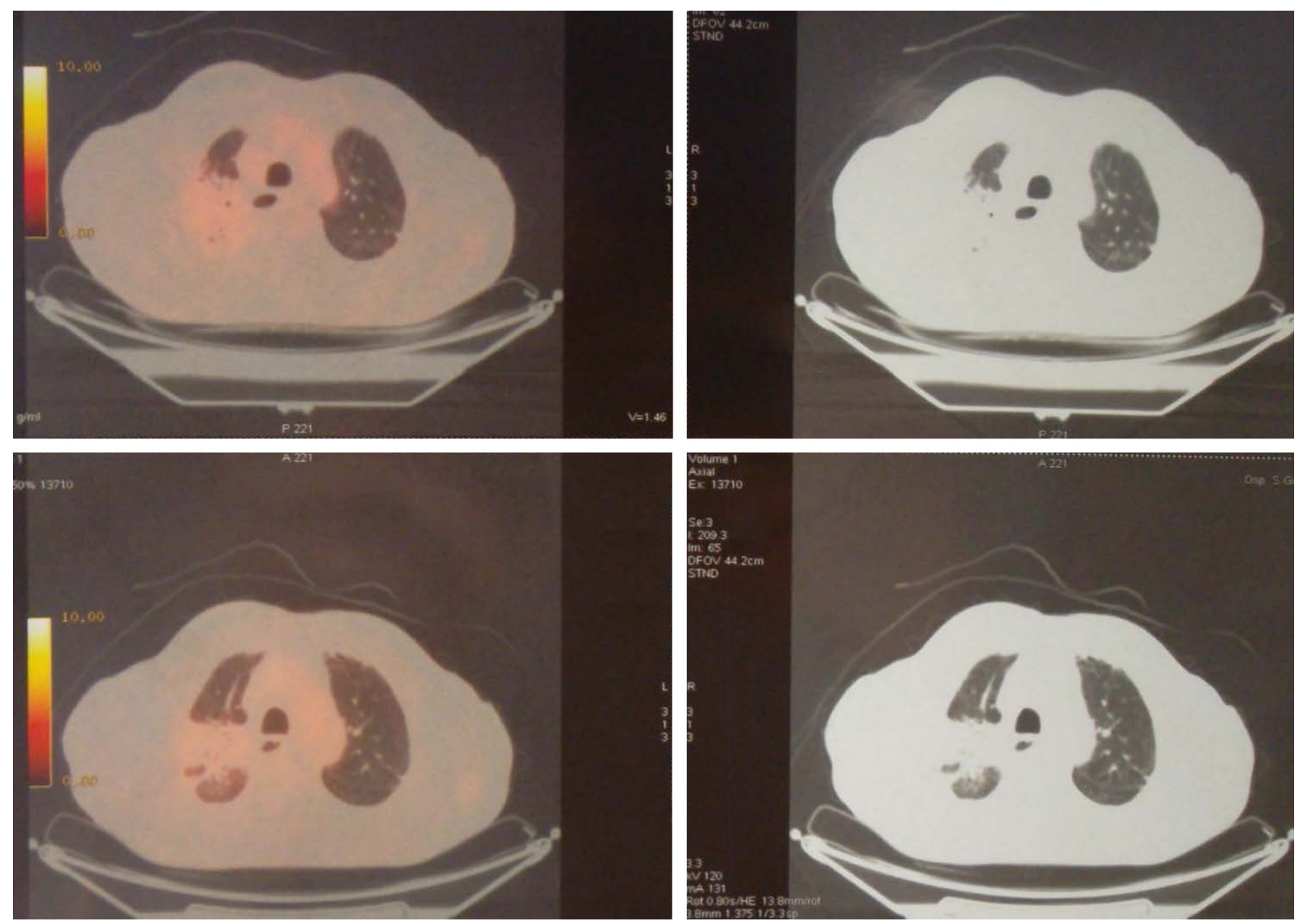

Figure 5 PET at last restaging after eight cycles of nivolumab.

Note: No fluorodeoxyglucose uptake and cavity with fibrosis in right lung is present, confirming a complete remission of disease.

Nivolumab, pembrolizumab, ipilimumab, tremelimumab, and durvalumab, at last, are all immune check point inhibitors capable of binding and inactivating the effects of the previously mentioned proteins, leading to immunogenic cancer cell death, with a safe toxicity profile. ${ }^{30,31}$ Moreover, preliminary reports suggest that factors such as PD-L1 expression, presence of TILs, and smoking, correlate with response to these therapies. ${ }^{32-34}$ All these factors were present in our patient.

Nivolumab is a fully humanized IgG4 monoclonal antibody $(\mathrm{mAb})$ that targets PD-1, and its successful use as a second line therapy in NSCLC has led to its definitive US Food and Drug Administration (FDA) approval as second line treatment for treatment-resistant patients, ${ }^{35,36}$ such as our patient. Despite the encouraging results, the response rate to immunotherapies is still low; in fact, positive outcomes have been recorded in approximately $17 \%$ to $19 \%$ of unselected patients, probably due to low immunogenic tumor expression in such cases..$^{37,38}$

This has led to the search for strategies to overcome this resistance, modifying the tumor immune microenvironment.
Data from many laboratories and sporadic reports indicate that local radiation therapy exerts immunostimulatory properties resulting in systemic, immune-mediated antitumor and distant anti-metastatic effects (abscopal effect) through increased tumor-antigen release and antigen-presenting cell cross-presentation, improved dendritic cell function, and enhanced T-cell priming. ${ }^{19}$ Preclinical and clinical reports have speculated regarding the concept of radiationinduced in situ vaccination as a result of radiation-induced expanded release of tumor-associated antigens and an amplified chemokine cascade (DAMPs), which causes the immunogenic death of cancer cells. ${ }^{39,40}$ As a result of mouse tumor models and clinical reports of more frequent abscopal responses in patients refractory to immunotherapy who received large sized hypofractionated RT during immunotherapy, the ability of RT to enhance antitumor $\mathrm{T}$ cell activity in synergy with immune check point inhibitors has been acknowledged..$^{41}$ Moreover, some authors have suggested that, in patients who would otherwise not respond to 
immune check point inhibitors, localized radiotherapy can induce activated tumor-specific T cells, thereby promoting response to these therapies. ${ }^{42}$ Anti-PD1 therapies are generally well-tolerated in clinical trials, however concerns exist about increased adverse events with radiotherapy and immunotherapy combinations, like high grade pneumonitis among NSCLC patients who previously received thoracic radiotherapy. The incidence of high grade pneumonitis in this patient population has been estimated as $4.1 \%$, suggesting the need for closer toxicity monitoring among patients who were previously treated with chest radiation. ${ }^{43}$ Interestingly, our patient developed pneumonitis after the completion of radiotherapy, before nivolumab treatment started. These argumentations suggest a role of radiotherapy-induced inflammatory activity in priming and homing of activated immune cells in the irradiated area leading to a favorable microenvironment more sensitive to the sequential anti-PDL1 activity. In support of this hypothesis, experimental data have shown how fractionated radiotherapy leads to an adaptive up-regulation of tumor cell PD-L1 expression that is dependent on CD8 T cells' production of IFN $\gamma$. This effect seems to attenuate the anticancer radiation immune response that can be overcome by the combination with mAB PD1-PDL1. ${ }^{44}$ Furthermore, Adams et al found that approximately 30\% of irradiated patients with NSCLC had a substantial increase of PD-L1 expression in circulating tumor cells 14-21 days after radiotherapy. In a sequential analysis of patients 2-4 months after completion of radiotherapy, nearly $87 \%$ of the PD-L1 expression remained unchanged from the T1 time point. ${ }^{45}$ These findings could be the rationale underlying the effect of nivolumab given 4 months after radiotherapy in our patient.

Going from the experimental to clinical data, currently, over a dozen clinical trials have evaluated the activity of antiPD1 antibodies in previously irradiated refractory NSCLC advanced stage patients. Chekmate 017 trial enrolling stage III-IV NSCLC pretreated patients reported that, compared to docetaxel, nivolumab achieved superior overall survival. ${ }^{46,47}$ It is curious that in this trial there was no correlation between PD-L1 expression and the benefit of nivolumab, and only one patient in the nivolumab arm (out of 272 enrolled patients) achieved a complete response. Nevertheless, from the study design it is not clear whether previously irradiated patients were included, and up to now no subgroup analysis data are available yet. An interesting suggestion regarding this concern has been put forward by the secondary analysis of the KEYNOTE-001 Phase I trial involving the group of previously irradiated NSCLC patients receiving pembrolizumab, which is another mAb that targets PD- $1 .^{48}$ In this analysis, patients receiving any radiotherapy to any site achieved a better and prolonged median progression-free survial and overall survival than non-irradiated patients. On the other hand, more recently the Pacific trial has reported exciting results with durvalumab vs placebo in a homogeneous population all pretreated with chemoradiation. ${ }^{49}$ Therefore, there are substantial data to support the hypothesis of the synergistic role of radiotherapy and sequential nivolumab, as shown in our patient in whom PD-L1 expression was positive but low. If so, the underlying mechanism could be as assumed by Demaria et al, who explained how radiotherapy is able to convert an immunogenic "cold" tumor into an immunogenic "hot" tumor, changing the game of immunotherapy. ${ }^{50}$ Certainly factors such as smoking, PD-L1 expression, and TILs have given an effective contribution to achieve this result.

\section{Conclusion}

Elderly patients with advanced NSCLC whose disease progresses or does not respond to first line chemotherapy and/or local radiotherapy have limited therapeutic options. The introduction of sequential or concomitant immunotherapy with conventional radiotherapy could be an effective therapeutic option to overcome cancer immune resistance and improve cancer-specific survival as emphasized by experimental models, ${ }^{44}$ Phase I-II clinical trials ${ }^{37-48}$ and sporadic reports, and more recently by a Phase III clinical trial. ${ }^{49}$

Apart from clinical trials, this case report confirms the experimental and preliminary data on the synergistic effects of nivolumab and radiotherapy in a heavily pretreated squamous cell lung cancer patient who had a poor response to radiotherapy. Robust Phase II-III clinical studies are required to better support these arguments. In fact, a Phase II safetyefficiency European Clinical Trial and Phase III RTOG 3505 trial providing nivolumab after standard chemoradiotherapy in stage III NSCLC patients have been designed, although, up until now they have not been open for enrollment. ${ }^{51,52}$

\section{Acknowledgments}

We would like to thank the Radiology Institute and Nuclear Medicine Institute of San Giuseppe Moscati Hospital for the availability of images.

\section{Author contributions}

All authors contributed toward data analysis, drafting and revising the paper and agree to be accountable for all aspects of the work. 


\section{Disclosure}

The authors report no conflicts of interest in this work.

\section{References}

1. Torre LA, Bray F, Siegel RL, et al. Global cancer statistics, 2012. CA Cancer J Clin. 2012;65(2):87-108.

2. Govindan R, Page N, Morgensztern D, et al. Changing epidemiology of small-cell lung cancer in the United States over the last 30 years: analysis of the surveillance, epidemiologic, and end results database. J Clin Oncol. 2006;24(28):4539-4544.

3. Penrod JR, Korytowsky B, Petrilla A, et al. Survival of U. S. Medicare patients with advanced non small cell lung cancer by line of therapy. J Clin Oncol. 2014;32(15 Suppl abstract).

4. Gridelli C, Maione P, Illiano A, et al. Cisplatin plus gemcitabine or vinorelbine for elderly patients with advanced non small-cell lung cancer: the MILES-2P studies. J Clin Oncol. 2007;25(29):4663-4669.

5. Socinski MA, Langer CJ, Okamoto I, et al. Safety and efficacy of weekly $\mathrm{nab}^{\circledR}$-paclitaxel in combination with carboplatin as first-line therapy in elderly patients with advanced non-small-cell lung cancer. Ann Oncol. 2013;24(2):314-321.

6. Shepherd FA, Dancey J, Ramlau R, et al. Prospective randomized trial of docetaxel versus best supportive care in patients with non-small-cell lung cancer previously treated with platinum-based chemotherapy. J Clin Oncol. 2000;18(10):2095-2103.

7. Kwint M, Walraven I, Burgers S, et al. Outcome of radical local treatment of non-small cell lung cancer patients with synchronous oligometastases. Lung Cancer. 2017;112:134-139.

8. Gajewski TF, Schreiber H, Fu YX, Yx F. Innate and adaptive immune cells in the tumor microenvironment. Nat Immunol. 2013;14(10): 1014-1022.

9. Demaria S, Formenti SC. Radiotherapy Effects on Anti-Tumor Immunity: Implications for Cancer Treatment. Front Oncol. 2013;3:128.

10. Kepp O, Tesniere A, Schlemmer F, et al. Immunogenic cell death modalities and their impact on cancer treatment. Apoptosis. 2009;14(4): 364-375.

11. Pardoll DM. The blockade of immune checkpoints in cancer immunotherapy. Nat Rev Cancer. 2012;12(4):252-264.

12. Chen DS, Irving BA, Hodi FS. Molecular pathways: next-generation immunotherapy - inhibiting programmed death-ligand 1 and programmed death-1. Clin Cancer Res. 2012;18(24):6580-6587.

13. Helissey C, Champiat S, Soria JC, Ghosh RK, Sharma A, Sharma N. Immune checkpoint inhibitors in advanced nonsmall cell lung cancer. Curr Opin Oncol. 2015;27(2):108-117.

14. Brahmer JR, Drake CG, Wollner I, et al. Phase I study of single-agent anti-programmed death-1 (MDX-1106) in refractory solid tumors: safety, clinical activity, pharmacodynamics, and immunologic correlates. J Clin Oncol. 2010;28(19):3167-3175.

15. Chen YB, Mu CY, Huang JA. Clinical significance of programmed death-1 ligand-1 expression in patients with non-small cell lung cancer: a 5-year-follow-up study. Tumori. 2012;98(6):751-755.

16. Velcheti V, Schalper KA, Carvajal DE, et al. Programmed death ligand-1 expression in non-small cell lung cancer. Lab Invest. 2014; 94(1):107-116.

17. Fridman WH, Pagès F, Sautès-Fridman C, Galon J. The immune contexture in human tumours: impact on clinical outcome. Nat Rev Cancer. 2012;12(4):298-306.

18. Liu H, Zhang T, Ye J, et al. Tumor-infiltrating lymphocytes predict response to chemotherapy in patients with advance non-small cell lung cancer. Cancer Immunol Immunother. 2012;61(10):1849-1856.

19. Formenti SC, Demaria S. Radiation therapy to convert the tumor into an in situ vaccine. Int J Radiat Oncol Biol Phys. 2012;84(4):879-880.

20. Formenti SC, Friedman K, Chao K, et al. Abscopal Response in Irradiated Patients: Results of a Proof of Principle Trial. Int J Radiat Oncol Biol Phys. 2008;72(1):S6-S7.

21. Vatner RE, Cooper BT, Vanpouille-Box C, Demaria S, Formenti SC. Combinations of immunotherapy and radiation in cancer therapy. Front Oncol. 2014;4:325.
22. Didkowska J, Wojciechowska U, Mańczuk M, Łobaszewski J. Lung cancer epidemiology: contemporary and future challenges worldwide. Ann Transl Med. 2016;4(8):150.

23. Chen YM, Perng RP, Shih JF, Whang-Peng J. A phase II randomized study of vinorelbine alone or with cisplatin against chemo-naïve inoperable non-small cell lung cancer in the elderly. Lung Cancer. 2008;61(2): 214-219.

24. Thomas L. On immunosurveillance in human cancer. Yale J Biol Med. 1982;55(3-4):329-333.

25. Dunn GP, Bruce AT, Ikeda H, Old LJ, Schreiber RD. Cancer immunoediting: from immunosurveillance to tumor escape. Nat Immunol. 2002; 3(11):991-998.

26. Dunn GP, Old LJ, Schreiber RD. The immunobiology of cancer immunosurveillance and immunoediting. Immunity. 2004;21(2):137-148.

27. Stagg J, Johnstone RW, Smyth MJ. From cancer immunosurveillance to cancer immunotherapy. Immunol Rev. 2007;220(1):82-101.

28. Postow MA, Callahan MK, Wolchok JD. Immune Checkpoint Blockade in Cancer Therapy. J Clin Oncol. 2015;33(17):1974-1982.

29. Zhou GW, Xiong Y, Chen S, Xia F, Li Q, Hu J. Anti-PD-1/PD-L1 antibody therapy for pretreated advanced nonsmall-cell lung cancer: A metaanalysis of randomized clinical trials. Medicine. 2016;95(35):35.

30. Dempke WC, Sellmann L, Fenchel K, Edvardsen K. Immunotherapies for NSCLC: Are We Cutting the Gordian Helix? Anticancer Res. 2015; 35(11):5745-5757.

31. Topalian SL, Hodi FS, Brahmer JR, et al. Safety, activity, and immune correlates of anti-PD-1 antibody in cancer. N Engl J Med. 2012;366(26): 2443-2454.

32. Schmidt LH, Kümmel A, Görlich D, et al. PD-1 and PD-L1 Expression in NSCLC Indicate a Favorable Prognosis in Defined Subgroups. PLoS One. 2015;10(8):e0136023-15.

33. Johnson SK, Kerr KM, Chapman AD, et al. Immune cell infiltrates and prognosis in primary carcinoma of the lung. Lung Cancer. 2000;27(1): 27-35.

34. Kinoshita T, Muramatsu R, Fujita T, et al. Prognostic value of tumorinfiltrating lymphocytes differs depending on histological type and smoking habit in completely resected non-small-cell lung cancer. Ann Oncol. 2016;27(11):2117-2123.

35. Rounds A, Kolesar J. Nivolumab for second-line treatment of metastatic squamous non-small-cell lung cancer. Am J Health Syst Pharm. 2015; 72(21):1851-1855

36. Rizvi NA, Mazières J, Planchard D, et al. Activity and safety of nivolumab, an anti-PD-1 immune checkpoint inhibitor, for patients with advanced, refractory squamous non-small-cell lung cancer (CheckMate 063): a phase 2, single-arm trial. Lancet Oncol. 2015;16(3):257-265.

37. Garon EB, Rizvi NA, Hui R, et al. Pembrolizumab for the treatment of non-small-cell lung cancer. N Engl J Med. 2015;372(21):2018-2028.

38. Gettinger SN, Horn L, Gandhi L, et al. Overall Survival and Long-Term Safety of Nivolumab (Anti-Programmed Death 1 Antibody, BMS936558, ONO-4538) in Patients With Previously Treated Advanced NonSmall-Cell Lung Cancer. J Clin Oncol. 2015;33(18):2004-2012.

39. Demaria S, Golden EB, Formenti SC. Role of Local Radiation Therapy in Cancer Immunotherapy. JAMA Oncol. 2015;1(9):1325-1332.

40. Reits EA, Hodge JW, Herberts CA, et al. Radiation modulates the peptide repertoire, enhances MHC class I expression, and induces successful antitumor immunotherapy. J Exp Med. 2006;203(5):1259-1271.

41. Sharabi AB, Lim M, Deweese TL, Drake CG. Radiation and checkpoint blockade immunotherapy: radiosensitisation and potential mechanisms of synergy. Lancet Oncol. 2015;16(13):e498-e509.

42. Vanpouille-Box C, Pilones KA, Wennerberg E, Formenti SC, Demaria S. In situ vaccination by radiotherapy to improve responses to anti-CTLA-4 treatment. Vaccine. 2015;33(51):7415-7422.

43. Nishino M, Giobbie-Hurder A, Hatabu H, Ramaiya NH, Hodi FS. Incidence of Programmed Cell Death 1 Inhibitor-Related Pneumonitis in Patients With Advanced Cancer: A Systematic Review and Metaanalysis. JAMA Oncol. 2016;2(12):1607-1616.

44. Dovedi SJ, Adlard AL, Lipowska-Bhalla G, et al. Acquired resistance to fractionated radiotherapy can be overcome by concurrent PD-L1 blockade. Cancer Res. 2014;74(19):5458-5468. 
45. Adams DL, Adams DK, He J, et al. Sequential Tracking of PD-L1 Expression and RAD50 Induction in Circulating Tumor and Stromal Cells of Lung Cancer Patients Undergoing Radiotherapy. Clin Cancer Res. 2017;23(19):5948-5958.

46. Brahmer J, Reckamp KL, Baas P, et al. Nivolumab versus Docetaxel in Advanced Squamous-Cell Non-Small-Cell Lung Cancer. N Engl J Med. 2015;373(2):123-135.

47. Horn L, Spigel DR, Vokes EE, et al. Nivolumab Versus Docetaxel in Previously Treated Patients With Advanced Non-Small-Cell Lung Cancer: Two-Year Outcomes From Two Randomized, Open-Label, Phase III Trials (CheckMate 017 and CheckMate 057). J Clin Oncol. 2017;35(35):3924-3933.

48. Shaverdian N, Lisberg AE, Bornazyan K, et al. Previous radiotherapy and the clinical activity and toxicity of pembrolizumab in the treatment of non-small-cell lung cancer: a secondary analysis of the KEYNOTE001 phase 1 trial. Lancet Oncol. 2017;18(7):895-903.
49. Antonia SJ, Villegas A, Daniel D, et al. Durvalumab after Chemoradiotherapy in Stage III Non-Small-Cell Lung Cancer. $N$ Engl J Med 2017;377(20):1919-1929.

50. Weichselbaum RR, Liang H, Deng L, Fu YX, Yx F. Radiotherapy and immunotherapy: a beneficial liaison? Nat Rev Clin Oncol. 2017 14(6):365-379.

51. Demaria S, Coleman CN, Formenti SC. Radiotherapy: Changing the Game in Immunotherapy. Trends Cancer. 2016;2(6):286-294.

52. Gerber DE, Urbanic JJ, Langer C, et al. Treatment Design and Rationale for a Randomized Trial of Cisplatin and Etoposide Plus Thoracic Radiotherapy Followed by Nivolumab or Placebo for Locally Advanced Non-Small-Cell Lung Cancer (RTOG 3505). Clin Lung Cancer. 2017 18(3):333-339.

\section{Publish your work in this journal}

OncoTargets and Therapy is an international, peer-reviewed, open access journal focusing on the pathological basis of all cancers, potential targets for therapy and treatment protocols employed to improve the management of cancer patients. The journal also focuses on the impact of management programs and new therapeutic agents and protocols on

\section{Dovepress}

patient perspectives such as quality of life, adherence and satisfaction. The manuscript management system is completely online and includes a very quick and fair peer-review system, which is all easy to use. Visit http://www.dovepress.com/testimonials.php to read real quotes from published authors.

Submit your manuscript here: http://www.dovepress.com/oncotargets-and-therapy-journal 